\title{
ultramicroscopy
}

Ultramicroscopy 61 (1995) 221-226

\section{Multi-detection and polarisation contrast in scanning near-field optical microscopy in reflection}

\author{
A. Jalocha, M.H.P. Moers, A.G.T. Ruiter, N.F. van Hulst * \\ Applied Optics Group, Department of Applied Physics and MESA Research Institute, University of Twente, P.O. Box 217, 7500 AE \\ Enschede, Netherlands
}

Received 9 May 1995; accepted 28 July 1995

\begin{abstract}
We present a scanning near-field optical microscope operating in reflection suitable for simultaneous bright field and fluorescence imaging. A non-coated pulled optical fibre is used in true reflection mode, i.e. both as emitter and collector. Beam splitters, dichroic mirrors and polarisers are combined to discriminate the different wavelength and polarisation signals. The distance between fibre and sample is controlled by shear force feedback. Bright field and fluorescence images with polarisation contrast of dielectric samples have been obtained with $200 \mathrm{~nm}$ lateral resolution. We show the fluorescence polarisation dependence with the molecular orientation in application to Langmuir-Blodgett thin film domains.
\end{abstract}

\section{Introduction}

Since the beginning of Scanning Near-field Optical Microscopy (SNOM) several schemes have been proposed [1]. Analogous to the situation in classical optical microscopy both transmission and reflection modes in different configurations have been developed [2-10], all with the aim to extract optical properties of the sample with a lateral resolution beyond the classical diffraction limit. Especially near-field fluorescence, as first demonstrated by Betzig et al. [11], is promising due to the combination of high sensitivity, strong background suppression and chemical specificity. Recently important progress in transmission near-field fluorescent microscopy of

\footnotetext{
* Corresponding author. Fax: +31 53 4891105; E-mail: N.F.vanHulst@tn.utwente.nl.
}

chemical and biological systems has been achieved [12-15], ultimately leading to single molecular detection and spectroscopy [16-19].

Bielefeld et al. [8] have shown first fluorescence images obtained in collection reflection mode in 1994. In this set-up the probe acts as a collector of the fluorescence signal, while the sample is externally illuminated. Here we describe a SNOM-reflection set-up where a non-coated fibre tip is used acting both as an emitter and a collector. Optical detection separates the bright field (resonant) and fluorescence signals. The probe to sample distance is controlled by a conventional shear force feedback system [20,21]. As a result bright field and fluorescence image and a force map are simultaneously obtained. In this paper applications to latex spheres and Langmuir-Blodgett (LB) films are presented. We demonstrate that reflected fluorescence polarisation contrast reveals characteristics of the molecular orientation in LB monolayer domains. 


\section{Microscope set-up}

The experimental set-up is schematically shown in Fig. 1. An $\mathrm{Ar}^{+}$laser beam $(\sim 100 \mathrm{~mW}, \lambda=514$ $\mathrm{nm}$ ) is launched into a multimode fibre. A polariser (P) controls the incoming beam polarisation. A commercial instrument (Sutter P2000) is used to pull the other extremity of the fibre which reaches less than $100 \mathrm{~nm}$ in size. The fibre tip is not coated and acts as an emitter and as a collector. A beam splitter (BS) combined with a photodiode (PD) allows the detection of the reflected beam $(\sim 1 \mu \mathrm{W})$ at the excitation wavelength (bright field signal). A dichroic mirror (DM, $\lambda>560 \mathrm{~nm}$ ) and a high pass filter (HPF, $\lambda>590 \mathrm{~nm}$ ) are used to separate the fluorescence from the excitation light coming from the sample. The fluorescence level is detected by a photomultiplier tube (PMT), where the polarisation is controlled by an additional analyser (A). This optical arrangement is suitable for the detection of polarised fluorescence and bright field signals simultaneously.

The tip-to-sample distance is regulated by a feedback on shear forces $[20,21]$. The fibre is oscillated laterally and its amplitude (typically $10 \mathrm{~nm}$ ) is monitored by differential detecting of the diffraction pattern of a laserdiode beam at the vibrating fibre. On approaching the sample the amplitude decreases due to "shear forces". The piezo voltage needed to keep the effective shear force signal at a constant level is recorded.

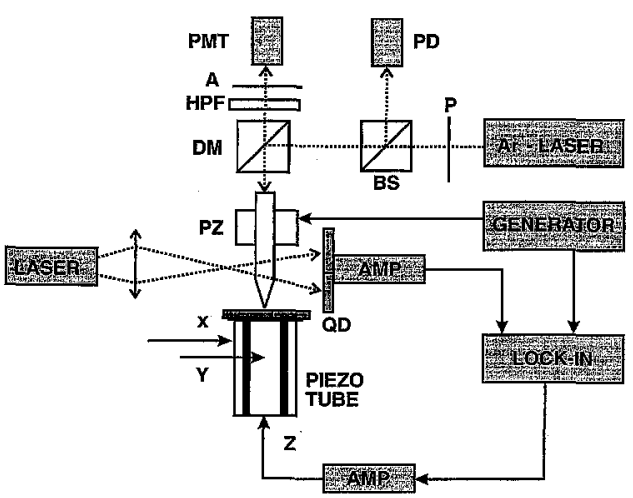

Fig. 1. SNOM-reflection set-up with simultaneous bright field, fluorescence and shear force detection. (A) Analyser, (BS) beam splitter, (DM) dichroic mirror, (HPF) high pass filter, (P) polariser, (PD) photodiode, (PZ) piezo actuator, and (QD) quadrant detector.
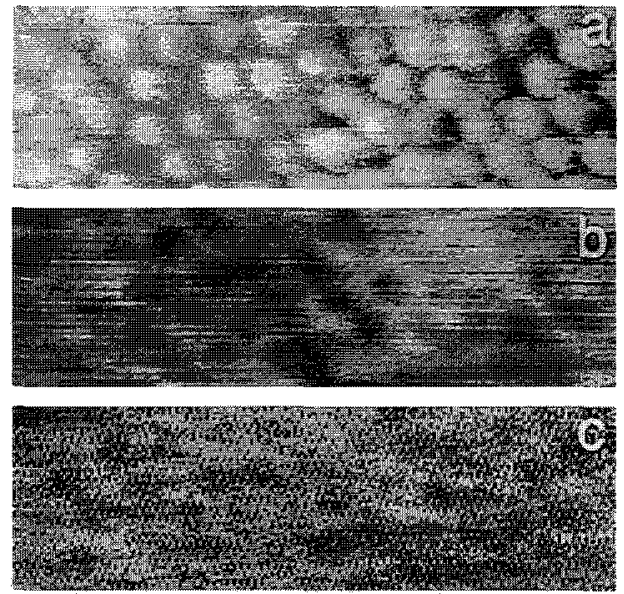

Fig. 2. Multilayer of $282 \mathrm{~nm}$ fluorescent latex spheres. Image size $3.4 \times 1.2 \mu \mathrm{m}^{2}$. (a) Shear force feedback signal, (b) corresponding bright field signal at $\lambda=514 \mathrm{~nm}$ and (c) corresponding red fluorescence signal. The three images are recorded simultaneously.

The sample is mounted on a piezo-electric tube allowing maximum $20 \times 20 \mu \mathrm{m}^{2}$ scan area. A personal computer generates the scan pattern and collects optical and force signals simultaneously. The data acquisition frequency is $300 \mathrm{~Hz}$, mainly limited by the bandwidth of the shear force feedback loop.

\section{Results}

Fig. 2 shows a $3.4 \times 1.2 \mu \mathrm{m}^{2}$ area of a multilayer of fluorescent latex spheres $\varnothing 282 \mathrm{~nm}$ in size (Molecular Probes, Eugene, OR, USA). The absorption band of this type of spheres (Nile red) is centred on $551 \mathrm{~nm}$ and the fluorescent emission is in the red around $620 \mathrm{~nm}$. The force image (a) displays clearly the hexagonal compacity of the layer. In the corresponding bright field image (b) the contour of each sphere is resolved. The sample topography induces changes in the optical signal and the spheres appear with different mutual contrast. The contrast in the fluorescence image (c) is much more clear. All spheres appear dark with a bright contour line. This result can be explained by the layer structure. The shear force feedback controls the tip-to-sample distance to an average constant value during the scan. The sample area contributing to the fluorescence 
signal is larger when the tip is placed in between the closely packed spheres than when it scans over the top of only one sphere. Thus the fluorescence signal detected by the probe is less on the top of a sphere making it to appear darker than its contour.

A Diethylene glycol Diamine PentacosaDiyonic Amide (DPDA) layer polymerised by ultra violet radiation has been investigated. A monolayer with a thickness of $6 \mathrm{~nm}$ was transferred onto an object glass slide by use of Langmuir-Blodgett techniques. The film consists of domains, varying from nanometers to several square micrometers in size [13,22]. All polymerised domains absorb in the green and fluoresce in the red. Each domain shows its specific absorption and emission moments as determined by its crystalline orientation. This growth particularity gives rise to an intense anisotropic fluorescence specific for each domain.

Results obtained on DPDA film are presented in
Fig. 3. The scan area is $8.0 \times 6.8 \mu \mathrm{m}^{2}$. In comparison of the simultaneously recorded shear force (a), bright field (b) and fluorescence (c) images several peculiarities are observed. The fluorescence image shows several fluorescent $(\sim 1 \mathrm{pW})$ domains of typically a few square microns, separated by non-fluorescent areas, where the glass substrate is. The shear force feedback image shows the reverse: high signal on the glass, low signal on the DPDA film, resulting in an inverted contrast with respect to the topography. The tip has to be about $14 \mathrm{~nm}$ closer to the polymer than to the glass surface to keep the effective shear force signal constant, despite the 6 $\mathrm{nm}$ height of the polymer. This observation is consistent with adhesion force imaging by van der Werf et al. [23] of the same LB film, showing that the adhesion interaction force with the hydrophobic LB film is much weaker than the interaction with the hydrophilic glass. Thus the chemical nature of the
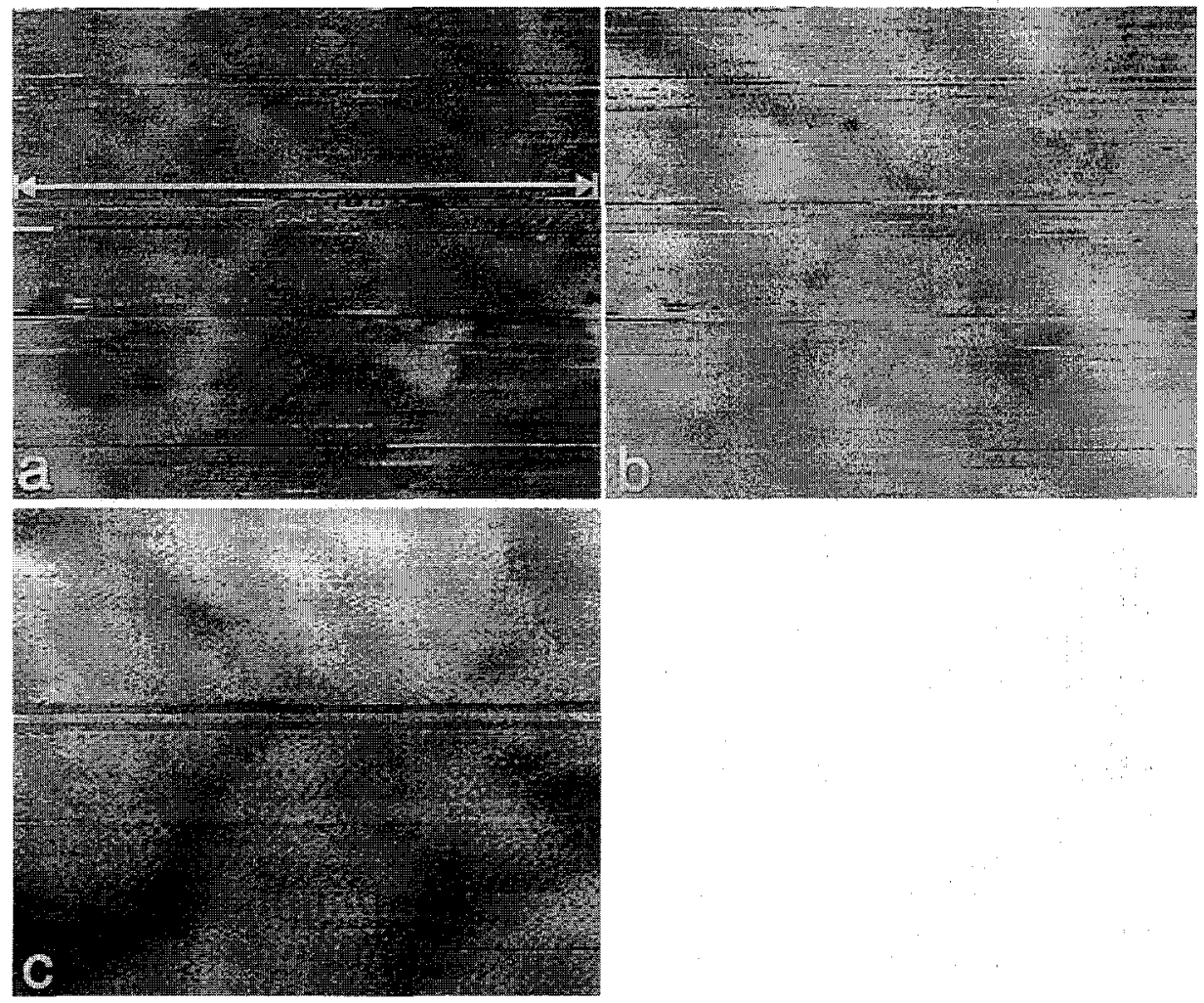

Fig. 3. DPDA Langmuir-Blodgett film, scan area $8 \times 6.8 \mu \mathrm{m}^{2}$, with corresponding shear force feedback (a), bright field (b) and fluorescence (c) image. 
sample is reflected in the shear force image. Finally the bright field image correlates strongly to the shear force image: on the glass areas the shear force feedback keeps the tip further from the surface resulting in reduced reflected light compared to the LB-film domains. The LB film absorbs about $10 \%$ of the light in transmission [13]; however, in bright field reflection this is not observed due to the dominant effect of topography and shear force regulation.

In Fig. 4 a fluorescence intensity profile of the DPDA film is plotted along the line indicated in Fig. $3 \mathrm{a}$. If we define the resolution as the width between maximum $-20 \%$ and minimum $+20 \%$, then averaged over several transitions we find about $200 \mathrm{~nm}$ lateral resolution.

Fig. 5 shows a scan of a different site of the DPDA film. The scan area is $10 \times 10 \mu \mathrm{m}^{2}$. The shear force image (a) displays several domains

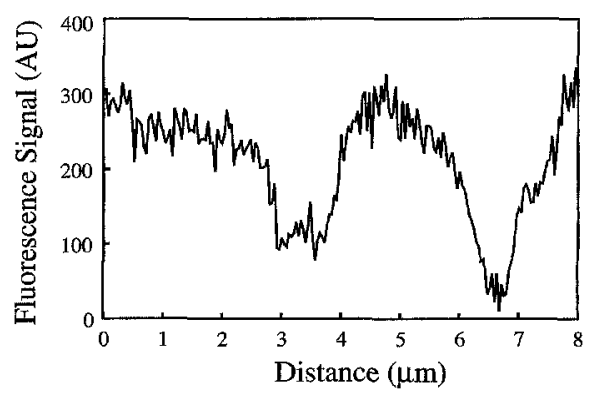

Fig. 4. Fluorescence line profile along the trace indicated in the shear force image Fig. 3a. The pixel size is $40 \mathrm{~nm}$. Fluorescence and non-fluorescence domains are distinguished with a resolution of $200 \mathrm{~nm}$.

smaller in size than those presented in Fig. 3. The polarisation of the fluorescence emission of each domain is analysed. Fluorescence images Figs. 5b,
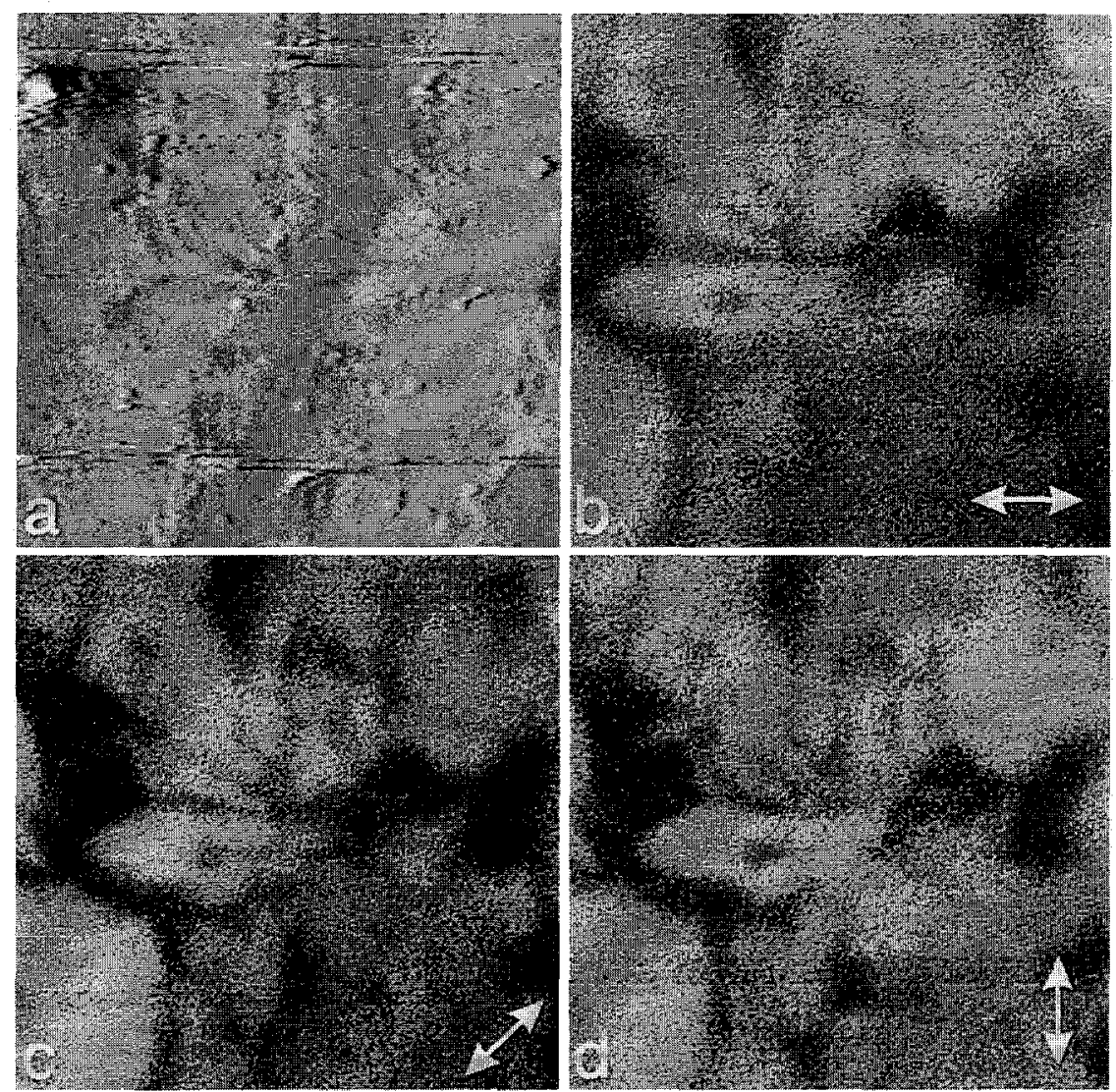

Fig. 5. DPDA Langmuir-Blodgett film, scan area $10 \times 10 \mu \mathrm{m}^{2}$. (a) Shear force feedback image displaying inverted topography. (b-d) Corresponding fluorescence images obtained with linearly polarised excitation at $0^{\circ}, 45^{\circ}$ and $90^{\circ}$, respectively, as indicated by the arrows. 
$5 \mathrm{c}$ and $5 \mathrm{~d}$ have been obtained by changing the orientation of the linear polarisation of both incident light and analyser at the photomultiplier tube. The linear polarisation in Fig. $5 \mathrm{~b}$ is $0^{\circ}$ (parallel to line scan direction) and rotated over $45^{\circ}$ and $90^{\circ}$ to realise Figs. 5c and 5d, respectively. One remarks that a slight shift between the images occurs, due to mechanical drift. The shear force image Fig. 5a corresponds to Fig. $5 c$. One observes the change of the relative fluorescence intensity of the domains on rotating the optical polarisation. Maximum fluorescence is obtained when the polarisation is parallel to absorption and emission moment of the polymer backbone in a given domain [13].

\section{Discussion and conclusions}

We presented a multi-detection Scanning NearField Optical Microscope in true reflection where the probe acts both as emitter and collector. The optical set-up allows to separate (polarised) fluorescence and bright field signals coming from the sample, as detected by the tip itself. Shear force feedback controls the distance between tip and sample surface. Thus three signals are recorded simultaneously.

The contrast in bright field images is affected by topography and shear force regulation, making them hard to interpret. Fluorescence images, especially of the flat LB monolayers, show a clear optical contrast with $200 \mathrm{~nm}$ lateral resolution. The anisotropy of the LB domains has been observed by fluorescence polarisation contrast, which enables determination of the orientation of individual domains.

We have observed "inverted topography" in shear force images of DPDA films. The "inversion" is attributed to the chemically specific nature of the shear forces, similar to the adhesion forces observed in "normal" atomic force microscopy in air. The sensitivity of shear forces for the hydrophobic/philic nature of the sample surface may have important applications for chemical mapping. On the other hand in near-field optical microscopy one should realise that "constant shear force signal" is not necessarily equal to "constant height", which may affect the near-field optical contrast. The chemical nature of shear forces is subject of our current research.
The fibre is not coated, which has the advantage of high brightness compared to metal coated aperture type probes [2], e.g. $\sim 1 \mu \mathrm{W}$ in reflection in our set-up. On the other hand the field confinement at the purely dielectric fibre probe is weak due to the absence of metal screening. Yet, in reflection a lateral resolution of about $200 \mathrm{~nm}$ is observed, probably due to preferential directional coupling in the reflection configuration. The combination of $1 \mu \mathrm{W}$ brightness and $200 \mathrm{~nm}$ resolution places this microscope in between the aperture type SNOM ( $1 \mathrm{nW}, 80$ $\mathrm{nm})$ and the conventional confocal microscope (10 $\mathrm{mW}, 300 \mathrm{~nm}$ ). In near-field studies where sufficient brightness is essential, e.g. Raman spectroscopy, our configuration is advantageous.

\section{Acknowledgements}

The LB films have been prepared by Uli Hoffmann and Hermann E. Gaub of the Technical University of Munich. This work was supported by the European Commission for Research (A.J. on contract: ERBCHGCT 920049), the European network for "Near-Field Optics and Nanotechnology" (N.F.v.H.) and the Dutch foundation FOM (M.H.P.M. and A.G.T.R.).

\section{References}

[1] D.W. Pohl and L. Novotny, J. Vac. Sci. Technol. B 12 (1994) 1441.

[2] E. Betzig, J.K. Trautman, T.D. Harris, J.S. Weiner and R.L. Kostelak, Science 251 (1991) 1468.

[3] T.L. Ferrell, S.L. Sharp and R.J. Warmack, Ultramicroscopy 42-44 (1992) 408.

[4] D. Courjon, J.M. Vigoureux, M. Spajer, K. Sarayeddine and S. Leblanc, Appl. Opt. 29 (1990) 3734.

[5] M. Spajer and A. Jalocha, in: Near Field Optics, Eds. D.W. Pohl and D. Courjon, NATO ASI Ser. E 242 (Kluwer, Dordrecht, 1993) p. 87.

[6] N.F. van Hulst, M.H.P. Moers and B. Bölger, J. Microscopy 171 (1993) 95.

[7] M. Garcia-Parajo, E. Cambril and Y. Chen, Appl. Phys. Lett. 65 (1994) 1498.

[8] H. Bielefeld, I. Hörsch, G. Krausch, M. Lux-Steiner, J. Mlynek and O. Marti, Appl. Phys. A 59 (1994) 103.

[9] M. Radmacher, P.E. Hillner and P.K. Hansma, Rev. Sci. Instrum. 65 (1994) 2737.

[10] J.A. Cline and M. Isaacson, Ultramicroscopy 57 (1995) 147. 
[11] E. Betzig, A. Lewis, A. Harootunian, M. Isaacson and E. Kratchmer, Biophys. J. 49 (1986) 269.

[12] E. Betzig, Bioimaging 1 (1993) 129.

[13] M.H.P. Moers, H.E. Gaub and N.F. van Hulst, Langmuir 10 (1994) 2774.

[14] A. Jalocha and N.F. van Hulst, Polarization contrast in fluorescence scanning near-ficld optical microscopy in reflection, in: J. Opt. Soc. Am. B. 12 (1995) 1577.

[15] M.H.P. Moers, A.G.T. Ruiter, A. Jalocha and N.F. van Hulst, Ultramicroscopy 61 (1995) 279.

[16] E. Betzig and R. Chichester, Science 262 (1993) 1422.

[17] J.K. Trautman, J.J. Macklin, L.E. Brus and E. Betzig, Nature 369 (1994) 40.
[18] W.P. Ambrose, P.M. Goodwin, J.C. Martin and R.A. Keller, Science 265 (1994) 364.

[19] W.E. Moerner, T. Plakhotnik, T. Irngartinger, U.P. Wild and D.W. Pohl, Phys. Rev. Lett. 73 (1994) 2764.

[20] R. Toledo-Crow, P.C. Yang, Y. Chen and M. Vaez-Iravani, Appl. Phys. Lett. 60 (1992) 2957.

[21] E. Betzig, P.L. Finn and J.S. Weiner, Appl. Phys. Lett. 60 (1992) 2484.

[22] B.M. Goettgens, R.W. Tillmann, M. Radmacher and H.E. Gaub, Langmuir 8 (1992) 1768.

[23] K.O. van der Werf, C.A.J. Putman, B.G. de Grooth and J. Greve, Appl. Phys. Lett. 65 (1994) 1195. 